\title{
Some Mappings on Operator Spaces
}

\author{
Guimei An \\ Department of Mathematics and LPMC, Nankai University, Tianjin, China \\ Email: angm@nankai.edu.cn \\ Received 7 February 2014; revised 7 March 2014; accepted 15 March 2014 \\ Copyright @ 2014 by author and Scientific Research Publishing Inc. \\ This work is licensed under the Creative Commons Attribution International License (CC BY). \\ http://creativecommons.org/licenses/by/4.0/ \\ (c) (i) Open Access
}

\begin{abstract}
We discuss two types of maps on operator spaces. Firstly, through example we show that there is an isometry on unit sphere of an operator space cannot be extended to be a complete isometry on the whole operator space. Secondly, we give a new characterization for complete isometry by the concept of approximate isometry.
\end{abstract}

\section{Keywords}

Unit Sphere, Approximate Isometry, Complete Isometry

\section{Introduction}

The theory of operator spaces is very recent. It was developed after Ruan's thesis (1988) by Effros and Ruan and Blecher and Paulsen and Pisier.

Theorem (Ruan) in [1] suppose that $X$ is a vector space, and that for each $n \in \mathbb{N}$ we are given a norm \|\|$_{n}$ on $M_{n}(X)$. Then $X$ is completely isometrically linearly isomorphic to a subspace of $B(H)$, for some Hilbert space $H$, if and only if conditions (R1) and (R2) above hold.

R1: $\|\alpha x \beta\|_{n} \leq\|\alpha\|\|x\|_{n}\|\beta\| \quad$ for $x \in M_{n}(V)$ and $\alpha, \beta \in M_{n}$,

R2: $\|x \oplus y\|_{n+m}=\max \left\{\|x\|_{n},\|y\|_{m}\right\} \quad$ for all $x \in M_{n}(V)$ and $y \in M_{m}(V)$.

In this new category, the objects remain Banach spaces but the morphisms become the completely bounded maps. In fact, the completely bounded maps appeared in the early 1980s following Stinespring's pioneering work (1955) and Arveson's fundamental results (1969) on completely positive maps see [1]-[4].

Definition 1 Let $X$ and $Y$ be operator spaces and that $u: X \rightarrow Y$ be a linear map. For a positive integer $n$, we write $u_{n}$ for the associated map $\left[x_{i j}\right] \mapsto\left[u\left(x_{i j}\right)\right]$ from $M_{n}(X)$ to $M_{n}(Y)$. This is often called the (nth) amplification of $u$, and may also be thought of as the map $I_{M_{n}} \otimes u$ on $M_{n} \otimes X$. A map $u$ is completely bounded (in short c.b.) if 


$$
\|u\|_{c b}:=\sup \left\{\left\|\left[u\left(x_{i j}\right)\right]\right\|\left\|_{n}:\right\|\left[x_{i j}\right] \|_{n} \leq 1 \text {, all } n \in \mathbb{N}\right\}<\infty .
$$

We denote by $C B(X, Y)$ the Banach space of all c.b. maps from $X$ into $Y$ equipped with the c.b. norm. If $u: X \rightarrow Y$ is c.b. map, we have

$$
\|u\| \leq\|u\|_{c b}
$$

and

$$
C B(X, Y) \subset B(X, Y) .
$$

The notion of isomorphism is replaced by that of "complete isomorphism". If $u: X \rightarrow Y$ is a completely bounded linear bijection, and if its inverse is completely bounded too, then we say that $u$ is complete isomorphism. In this case, we say that $X$ and $Y$ are completely isomorphic and we write " $X \approx Y$ as operator spaces". Similarly, if $u_{n}$ is an isometry for any $n \in \mathbb{N}$, then we say that $u$ is complete isometry. We often identify two operator spaces $X$ and $Y$ if they are completely isometrically isomorphic. In this case we often write " $X \cong Y$ as operator spaces".

In 1932 [5], Mazur and Ulam got the famous theorem that a surjective isometry between two real normed spaces must be linear up to translation, which started the study of the theory of isometry. Many researchers have tried to generalize it. In 1972 Mankiewicz [6] considered the extension problem for isometries and every surjective isometry between the open connected subsets of two normed spaces can be extended to a surjective affine isometry on the whole space. This result can be seen as local Mazur-Ulam Theorem. From this result, we get easily that a map's property on unit ball of a normed space determines the relationship between distance preserving and linearity. In 1987, D.Tingley raised the following problem (the isometric extension problem) in [7]:

Problem. Suppose that $V_{0}: S_{1}(X) \rightarrow S_{1}(F)$ is a surjective isometric mapping, does there exist a linear isometric mapping $V: X \rightarrow Y$ such that $\left.V\right|_{S_{1}(X)}=V_{0}$ ?

D. Tingley, who is the first one to study the problem, gave some results in [7] under the condition that $X$ and $Y$ are finite dimensional spaces. More precisely, the answer can be formulated as follows: Suppose that $V_{0}: S_{1}(X) \rightarrow S_{1}(Y)$ is a surjective isometric mapping, then $V_{0}(-x)=-V_{0}(x)$, i.e. $V_{0}$ is an odd mapping. In the complex spaces, the answer to Tingleys problem is negative. For example, we take $X=Y=\mathbb{C}$ (complex plane) and $V(x)=\tilde{x}$. Some affirmative results have been obtained between classical real Banach spaces, which had been shown in [8]-[12]. The recent development on this problem you can find in [13]. We ask can we discuss the isometric extension problem in operator space category? Through example, we give some results on it.

\section{Some Examples about Isometric Extension Problem on Operator Spaces}

Theorem Let $X$ be $R$ (resp. $C, O H, M I N(H), \operatorname{MAX}(H),(\operatorname{MIN}(H), M A X(H))_{\theta}$, etc.). Let $V_{0}: S_{1}(X) \rightarrow S_{1}(X)$ be a 1 -Lip operator. Then $V_{0}$ can be extended to a linearly complete isometry on $X$.

Proof. By [11], $V_{0}$ can be linearly extended to the whole Hilbert space. Since $X$ are homogeneous operator space, the the result can be got directly.

How about the result on non-homogeneous operator spaces?

Theorem There exists an isometry $V_{0}: S_{1}\left(H_{c} \otimes H_{r}\right) \rightarrow S_{1}\left(H_{c} \otimes H_{r}\right)$ such that there is no complete isometry $V$ on the whole space $H_{c} \otimes H_{r}$ satisfying $\left.V\right|_{S_{1}\left(H_{c} \otimes H_{r}\right)}=V_{0}$.

Proof. Let $\left\{e_{i}=(0, \cdots, 0,1,0 \cdots, 0)\right\}_{i=1}^{n}$ be the basis of $\mathbb{C}^{n}$. To express conveniently, we denote $\left\{e_{i}\right\}$ $(1 \leq i \leq n)$ to be the basis of the $n$-dimensional column Hilbert operator space $H_{c}$ and $\left\{f_{k}\right\} \quad(1 \leq k \leq n)$ be the basis of the $n$-dimensional row Hilbert operator space $H_{r}$. Then $\left\{e_{i} \otimes f_{k}\right\}$ constructs a basis of $H_{c} \otimes H_{r}$. Give the $n^{2}$ elements an order defined as

$$
h_{i}=\left\{\begin{array}{lc}
e_{1} \otimes f_{i} & (1 \leq i \leq n) ; \\
e_{1+k} \otimes f_{1} & (i=n+k, 1 \leq k \leq n-1) ; \\
\text { others } & (i \geq 2 n) .
\end{array}\right.
$$


Let $V\left(h_{1}\right)=h_{1}, V\left(h_{i}=h_{n+i-1}\right),(2 \leq i \leq n)$ and $V$ maps other elements $\left\{e_{i} \otimes f_{j}\right\}_{i, j=1}^{n}-\left\{e_{1} \otimes f_{i}\right\}_{i=1}^{n}$ of the basis to $\left\{e_{i} \otimes f_{j}\right\}_{i, j=1}^{n}-\left\{e_{i} \otimes f_{1}\right\}_{i=1}^{n}$. So $V$ forms a permutation among the basis of $H_{c} \otimes H_{r}$. By the isometric theory on Hilbert space, $V$ is an isometry on the Banach space $H_{c} \otimes H_{r}$. Of course let $V_{0}=\left.V\right|_{H_{c} \otimes H_{r}}$ is an isometry between unit sphere of $H_{c} \otimes H_{r}$. But $V$ is not complete isometry on $H_{c} \otimes H_{r}$.

Indeed, for $x=\left(x_{i j}\right) \in M_{n}\left(H_{c} \otimes H_{r}\right)$ where

$$
x=\left(\begin{array}{cccc}
e_{1} \otimes f_{1} & 0 & \cdots & 0 \\
e_{1} \otimes f_{2} & 0 & \cdots & 0 \\
\cdots & 0 & \cdots & 0 \\
e_{1} \otimes f_{i} & 0 & \cdots & 0 \\
\cdots & 0 & \cdots & 0 \\
e_{1} \otimes f_{n} & 0 & \cdots & 0
\end{array}\right) .
$$

Then

$$
V_{n}(x)=\left(\begin{array}{cccc}
e_{1} \otimes f_{1} & 0 & \cdots & 0 \\
e_{2} \otimes f_{1} & 0 & \cdots & 0 \\
\cdots & 0 & \cdots & 0 \\
e_{i} \otimes f_{1} & 0 & \cdots & 0 \\
\cdots & 0 & \cdots & 0 \\
e_{n} \otimes f_{1} & 0 & \cdots & 0
\end{array}\right) .
$$

It is easy to calculate that $\|x\|_{c}=1$ but $\left\|V_{n}(x)\right\|_{r}=\sqrt{n}$. So $V$ is not complete isometry.

\section{On Stability of $\epsilon$-Isometries of Operator Spaces}

The study on the stability of $\epsilon$-isometry is from Banach Space Theory.

Let $X, Y$ be two Banach spaces and $\epsilon \geq 0$. A mapping $f: X \rightarrow Y$ is said to be an $\epsilon$-isometry provided

$$
\|f(x)-f(y)\|-\|x-y\|<\epsilon \text {, for all } x, y \in X .
$$

In 1945, Hyers and Ulam proposed the following question: whether for every surjective $\epsilon$-isometry $f: X \rightarrow Y$ with $f(0)=0$ there exist a surjective linear isometry $U: X \rightarrow Y$ and $\gamma>0$ such that $\|f(x)-U x\| \leq \gamma \epsilon$ for all $x \in X$. After many efforts of a number of mathematicians (see, for instance [14]-[19]) Omladi $\breve{c}$ and $\breve{S}$ emrl gave the sharp estimate that $\gamma=2$ in 1995 (see [20]).

In this section, we ask whether we can discuss the the stability of $\epsilon$-isometry in operator space category? We try to propose a question and give a little answer.

Firstly, we get the following result.

Theorem Let $X$ and $Y$ be operator spaces. If a mapping $T: X \rightarrow Y$ satisfies that for any $n \in \mathbb{N}$,

$$
\left\|T_{n}(x)-T_{n}(y)\right\|_{n}-\|x-y\|_{n} \mid \leq \epsilon,\left(\forall x, y \in M_{n}(X)\right),
$$

then $T$ is an isometry.

Proof. For any $n \in \mathbb{N}, T_{n}: M_{n}(X) \rightarrow M_{n}(Y)$. Let $x, y \in X$. Specially, we put $x_{i j} \equiv x, y_{i j} \equiv y,(1 \leq i, j \leq n)$, then $\left[x_{i j}\right],\left[y_{i j}\right] \in M_{n}(X)$ and we have

$$
T_{n}\left[x_{i j}\right]-T_{n}\left[y_{i j}\right]=(T x-T y) \otimes\left[\begin{array}{ccc}
1 & \cdots & 1 \\
\vdots & \ddots & \vdots \\
1 & \cdots & 1
\end{array}\right],
$$

Since 


$$
\lim _{n \rightarrow \infty}\left\|\left[\begin{array}{ccc}
1 & \cdots & 1 \\
\vdots & \ddots & \vdots \\
1 & \cdots & 1
\end{array}\right]\right\|_{n}=\infty
$$

we get $\|T x-T y\|-\|x-y\| \leq \epsilon\left\|\left[\begin{array}{ccc}1 & \cdots & 1 \\ \vdots & \ddots & \vdots \\ 1 & \cdots & 1\end{array}\right]\right\|^{-1}$.

So $\|T x-T y\|=\|x-y\|$ holds for any $x, y \in X . T$ is an isometry.

\section{Acknowledgements}

A part of this work was completed when I visited the University of Illinois, Urbana. I would like to thank Professor Zhong-Jin Ruan for the invitation and great help.

The author also expresses his appreciation to Dr. Liu Rui for many very helpful comments regarding the stability of $\epsilon$-isometry in operator space category.

\section{Funding}

This work is supported by the NSFC grant 11101220, 11271199 and the Fundamental Research Funds for the Central Universities.

\section{References}

[1] Effros, E.G. and Ruan, Z-J. (2000) Operator Spaces. In: London Mathematical Society Monographs New Series 23, The Clarendon Press, Oxford University Press, New York.

[2] An, G., Lee, J.-J. and Ruan, Z.-J. (2010) On p-Approximation Properties for p-Operator Spaces. Journal of Functional Analysis, 259, 933-974. http://dx.doi.org/10.1016/j.jfa.2010.04.007

[3] Pisier, G. (1990) Completely Bounded Maps between Sets of Banach Space Operators. Indiana University Mathematics Journal, 39, 249-277. http://dx.doi.org/10.1512/iumj.1990.39.39014

[4] Pisier, G. (2003) An Introduction to the Theory of Operator Spaces. In: London Mathematical Society Lecture Note Series 294, Cambridge University Press, Cambridge.

[5] Mazur, S. and Ulam, S. (1932) Sur less transformations isométriques d'espaces vectoriels normés. Comptes Rendus de l'Académie des Sciences de Paris, 194, 946-948.

[6] Mankiewicz, P. (1972) On Extension of Isometries in Normed Linear Spaces. Bulletin de l Académie Polonaise des Sciences, Série des Sciences Mathématiques, Astronomiques, et Physiques, 20, 367-371.

[7] Tingley, D. (1987) Isometries of the Unit Sphere. Geometriae Dedicata, 22, 371-378. http://dx.doi.org/10.1007/BF00147942

[8] Ding, G.G. (2003) The Isometric Extension Problem in the Unit Spheres of $l_{p}(\Gamma)(p>1)$ Type Spaces. Science in China, Series A, 46, 333-338.

[9] Ding, G.G. (2004) The Representation Theorem of onto Isometric Mappings between Two Unit Spheres of $l_{1}(\Gamma)$ Type Spaces and the Application to Isometric Extension Problem. Acta Mathematica Sinica, English Series, 20, 10891094. http://dx.doi.org/10.1007/s10114-004-0447-7

[10] Ding, G.G. (2004) The Representation of onto Isometric Mappings between Two Spheres of $l^{\infty}$-Type Spaces and the Application on Isometric Extension Problem. Science in China Series A, 47, 722-729. http://dx.doi.org/10.1360/03ys0049

[11] Ding, G.G. (2002) The 1-Lipschitz Mapping between the Unit Spheres of Two Hilbert Spaces Can Be Extended to a Real Linear Isometry of the Whole Space. Science in China Series A, 45, 479-483. http://dx.doi.org/10.1007/BF02872336

[12] Fang, X.N. and Wang, J.H. (2006) Extension of Isometries between the Unit Spheres of Normed Space E and C( $\Omega$ ), Acta Mathematica Sinica, English Series, 22, 1819-1824. http://dx.doi.org/10.1007/s10114-005-0725-z

[13] Ding, G.G. (2009) On Isometric Extension Problem between Two Unit Spheres. Science in China Series A, 52, 20692083. http://dx.doi.org/10.1007/s11425-009-0156-x 
[14] Bourgin, D.G. (1946) Approximate Isometries. Bulletin of the American Mathematical Society, 52, 704-714. http://dx.doi.org/10.1090/S0002-9904-1946-08638-3

[15] Bourgin, D.G. (1975) Approximate on Finite Dimensional Banach Spaces. Transactions of the American Mathematical Society, 207, 309-328.

[16] Figiel, T. (1968) On Non Linear Isometric Embedding of Normed Linear Spaces. Bulletin de l Académie Polonaise des Sciences, Série des Sciences Mathématiques, Astronomiques, et Physiques, 16, 185-188.

[17] Gruber, P.M. (1978) Stability of Isometries. Transactions of the American Mathematical Society, 245, $263-277$. http://dx.doi.org/10.1090/S0002-9947-1978-0511409-2

[18] Gevirtz, J. (1983) Stability of Isometries on Banach Spaces. Proceedings of the American Mathematical Society, 89, 633-636. http://dx.doi.org/10.1090/S0002-9939-1983-0718987-6

[19] Ulam, S.M. and Hyers, D.H. (1945) On Approximate Isometries. Bulletin of the American Mathematical Society, 51, 288-292.

[20] Omladic, M. and Semrl, P. (1995) On Non Linear Perturbations of Isometries. Mathematische Annalen, 303, 617-628. http://dx.doi.org/10.1007/BF01461008 